\title{
Characterization study of a thermal oil-based carbon black solar
}

\author{
nanofluid
}

\author{
A. Gimeno-Furio ${ }^{1 *}$, L. Hernandez ${ }^{1}$, N. Navarrete ${ }^{1}$, R. Mondragon ${ }^{1}$ \\ ${ }^{1}$ Department of Mechanical Engineering and Construction, Universitat Jaume I, Castellón, \\ Spain \\ *Corresponding author: afurio@uji.es
}

Email addresses:

Leonor Hernández: Ihernand@uji.es

Alexandra Gimeno: afurio@uji.es

Nuria Navarrete: nnavarre@uji.es

Rosa Mondragón: mondragn@uji.es 


\section{Abstract}

Carbon nanoparticles are very useful in solar thermal applications, since they absorb much of the solar spectrum, are cheap and have excellent optical properties. Carbon nanoparticlesthermal oil-based nanofluid was prepared using two-step method with diphenyl sulfone as surfactant to achieve that nanoparticles remain suspended even at high temperatures. The size particle distribution was studied using two Dynamic Light Scattering systems at room and high temperature and also evaluated before and after exposing the nanofluid to a thermal treatment so that conditions closer to those in real applications were replicated. Moreover, the morphological changes due to the thermal treatment were observed with Transmission Electron Microscopy. Finally, the optical properties as the ballistic transmittance, absorption coefficient and scattering albedo of the base fluid as well as of the nanofluid were measured using a spectrophotometer with and without integrating sphere. The results of this study contribute to the knowledge about these solar nanofluids that are promising alternatives to the conventional solar collectors.

Key words: solar absorbers; carbon black; thermal oil; absorption coefficient; nanofluids. 


\section{Introduction}

Concentrated Solar Power (CSP) plants, which convert the sun radiation to heat, are widely used in applications ranging from hot water supply to large-scale electricity production. Clean energy demand is growing continuously and solar thermal processes are expected to be a potential source of energy in the future [1]. A new idea of the solar thermal collectors was described by [2], introducing the concept in which solar radiation is absorbed and transported by the same working fluid. The majority of the heat transfer fluids (HTF) used (water, glycols, oils, etc.) are transparent for most part of the solar spectrum [3], which allows that adding some additives to the working fluid can enhance their absorption properties. One of the most frequently used additives is an organic compound, India Ink, but it presents some drawbacks when working at high temperature. It experiences light- and temperature induced degradation [4] as well as other organic and inorganic compounds, which can influence negatively in the absorption and can produce fouling of surfaces over time. Due to the limitations, that using dyes present, the addition of nanoparticles appears as a potential alternative to improve the absorption properties of the heat transfer fluid. The properties that make nanofluids an appropriate candidate for direct solar thermal energy absorption applications are the clogging and fouling avoidance when they pass through pumps and pipes [5].

Nanofluids, which are suspensions with nanoparticles suspended whose sizes ranges from 10 $\mathrm{nm}$ to $100 \mathrm{~nm}$, have obtained a lot of consideration over the years. Recent works have demonstrated that more promising applications of nanofluids could be utilising their optical properties, which are highly adjustable [6- 9]. Indeed, the use of nanofluids as both solar collector and heat transfer fluids is intended to improve efficiencies and reduce costs in solar thermal systems.

One of the main obstacles when using nanofluids, is to reach that nanoparticles remain suspended in the base fluid. The properties attributed to the nanofluids are consequence of 
the nanometer size and homogeneous dispersion of the particles, and then if the nanoparticles agglomerate or settle, properties mentioned above will be no longer associated to the nanofluid. Among others, the most common method to obtain a stable nanofluid is through the use of surfactants [10]. Unfortunately, most surfactants suffer chemical degradation and can loss efficacy at low temperatures as $70^{\circ} \mathrm{C}[11]$. Indeed, some techniques are used to analyse the particle size distribution of the nanofluid at both room and high temperature. Usually, Dynamic Light Scattering (DLS) technique is employed to measure the size of the nanofluid's particles through the backscattered light that varies depending on the size of the particles. Particle size distribution can be measured over the time and comparing the results, it can be determined if the colloids have been altered.

In this study, carbon black (CB) nanoparticles were added to Therminol 66 thermal oil (TH66) using dyphenil sulfone (DS) as surfactant. The nanoparticles have been selected due to their black absorption spectrum[12-14], the base fluid due to their extended use as HTF $[15,16]$ and the surfactant because its good results at high temperatures [17].

The aim of this work is to quantitatively study the high-temperature behaviour of $\mathrm{CB}$ nanofluids in direct solar absorption applications. Two different DLS systems were used to evaluate the particle size distributions of the $C B$ nanofluid at different temperatures. Moreover, the samples were thermally treated at $85^{\circ} \mathrm{C}$ during 30 minutes and they were measured again with DLS and also examined with electron microscopy to get a more detailed information about the effect of the thermal treatment. Also, optical tests was carried out to determine different optical properties to evaluate the suitability of the studied nanofluid as direct solar absorber. 


\section{Materials and methods}

\subsection{Synthesis of nanofluids}

The base fluid used herein was TH66 (Solutia Inc.). It consists of a hydrogenated terphenyl with proper thermal properties, which allows its use for high-temperature working conditions. CB nanoparticles (ELFTEX 570, Cabot Corporation) were selected for their high solar absorption ability and because their structure is not affected by high temperatures. According to the manufacturer, they consist of spherical amorphous carbon particles with a primary diameter of $10 \mathrm{~nm}$. The surfactant used was diphenyl sulfone (DS, Sigma Aldrich Co. Ltd.) and it was selected for its chemical affinity to thermal oils and its effective thermal behaviour at high temperature.

Nanofluid was prepared by adding CB nanoparticles to the base fluid at a $0.0016 \%$ weight concentration by the two-step method. Firstly, the stabiliser was mixed and dispersed in TH66 by an ultrasound probe (Sonopuls HD2200, Bandelin) for $1 \mathrm{~min}$. Then the CB nanoparticles were added and dispersed by an ultrasound probe for $1 \mathrm{~min}$. The nanoparticle concentration was selected so that the transmission of the resulting nanofluid lowered by $20 \%$ of the base thermal oil values. The selected surfactant/nanoparticle ratio was 1:1 based on previous works $[17,18]$

\subsection{Morphology}

Samples were observed in the Transmission Electron Microscopy (TEM) before and after the thermal treatment at $85^{\circ} \mathrm{C}$ for 30 minutes in a stove (Digitronic 2005141, J.P. Selecta, S.A.) in order to characterise possible changes in the nanoparticles.

\subsection{Experimental facilities}

\subsubsection{Zetasizer Nano (Malvern Instruments)}


One of the equipment used for measuring the size particle distribution is Zetasizer Nano (Malvern Instruments) which is commonly used in literature to measure particle size distributions of nanofluids by means of DLS. The main components of the equipment are a laser, that illuminates the cell containing the sample, and a detector that acquires the intensity of the light scattered by the nanoparticles suspended. Everything is enclosed in the system, and the diameter is obtained from the intensity of the backscattered light at $173^{\circ}$. Moreover, the Zetasizer Nano incorporates a heating system that allows measures at high temperature up to $90^{\circ} \mathrm{C}$, which allows evaluating particle size distributions at different temperatures.

\subsubsection{VASCO FLEX (Cordouan Technologies)}

VASCO FLEX particle size analyser (Cordouan Technologies) is a new system to determine particle size distributions. The principal difference between these two DLS devices is that in the new one the laser head is external and contains the light source and the receiver. In this system, the light registered corresponds to backscattering with an angle of $170^{\circ}$. Since the device is external, the temperature range of the measurement only depends on the design of the cuvette were the sample is located. In this case, the cuvette is made of aluminium with two fused quartz windows on the sides with a $20 \mathrm{ml}$ sample volume. A heating ring surround the cuvette, and two K-thermocouples are used to control the temperature on the wall of the cuvette and inside the fluid. The maximum working temperature of this system is $300^{\circ} \mathrm{C}$.

A more detailed scheme of the cuvette and general experimental set-up can be observed in Figure 1.

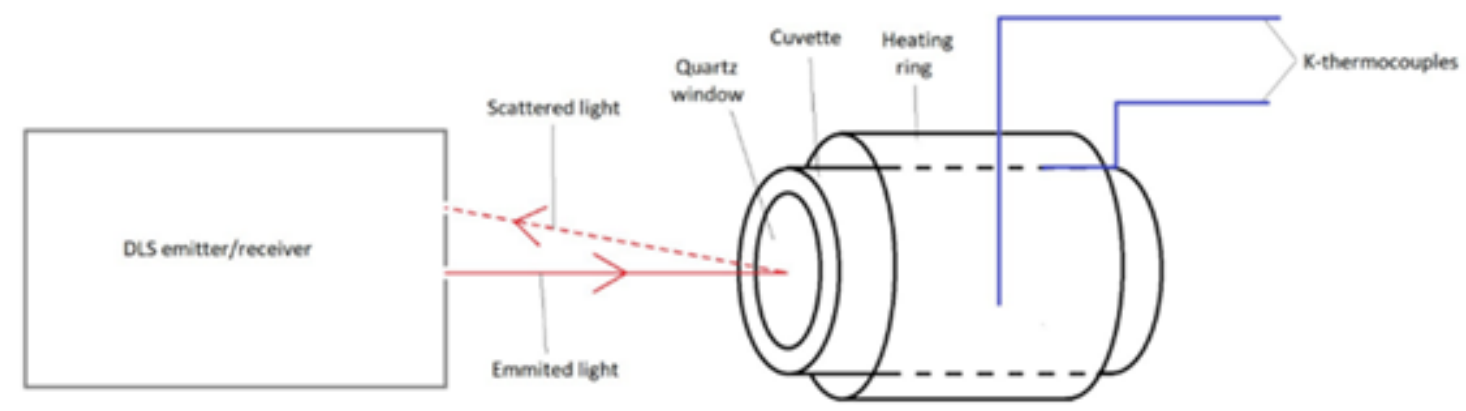


Figure 1 Experimental set-up of VASCO FLEX system.

\subsubsection{CARY 500 Spectrophotometer (Varian Devices)}

CARY 500 is a closed spectrophotometer and its main elements are: a source of light, which consists of a Tungsten lamp, a receiver located in front of the light emitter and a cuvette holder where the sample to be analysed is placed. The operating principle of this system is to measure the intensity of the light that it transmitted (ballistic transmittance $(\mathrm{T})$ ) by the fluid, evaluating the intensity of the sample beam and the light received after passing through the sample. The measured ballistic transmittance in the case, includes the absorbance but also the scattering of the sample.

In Figure 2 a detailed scheme of the system is represented.

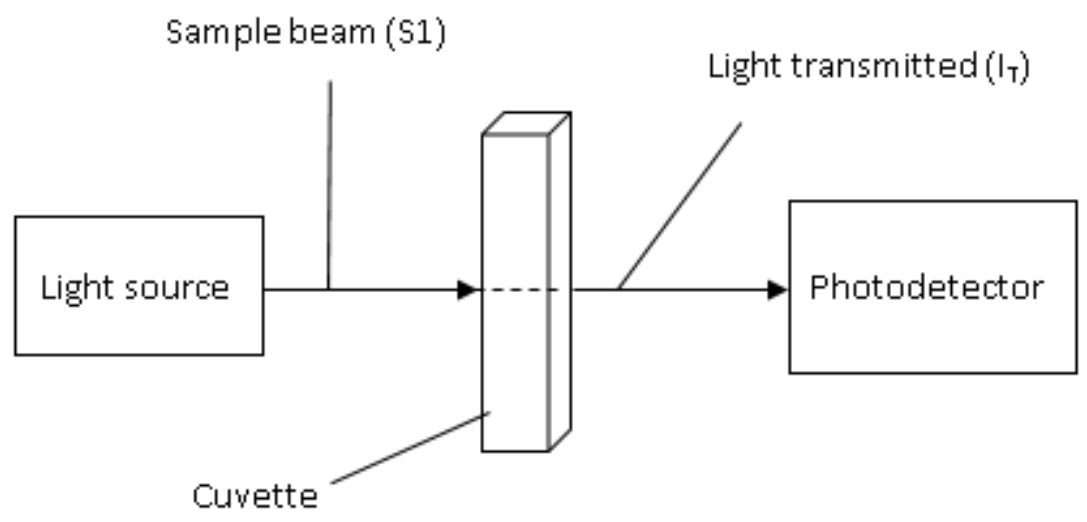

Figure 2 A schematic of the spectrophotometer CARY 500 without integrating sphere.

To be able to measure the absorption of the sample, an integrating sphere is attached to the spectrophotometer. Figure 3 illustrates the detection geometry of the integrating sphere. Two laser beams are directed to the sphere, one directed to the sample itself and a second serving as reference. Once the beams enter the sphere, they impact onto its inner Teflon surface and suffer many internal reflections before being captured by the photodetector (D) at the top of the integrating sphere. Note that the reference beam enters the sphere through a diffusing 
window (R1) to reduce the losses that would arise from a direct reflection at R2. This beam serves to monitor the laser power changes over the measurement time required for a full wavelength scan. The sample beam enters the integrating sphere through a hole in port S1 and impinges directly onto the port S2 (which can be in a tilted as in Figure 3 or not tiltled position). In practice, the sample can be placed in any of the two ports (S1 or S2) depending on the desired property to be measured. In order to obtain the absorption spectrum, two measurements were needed.

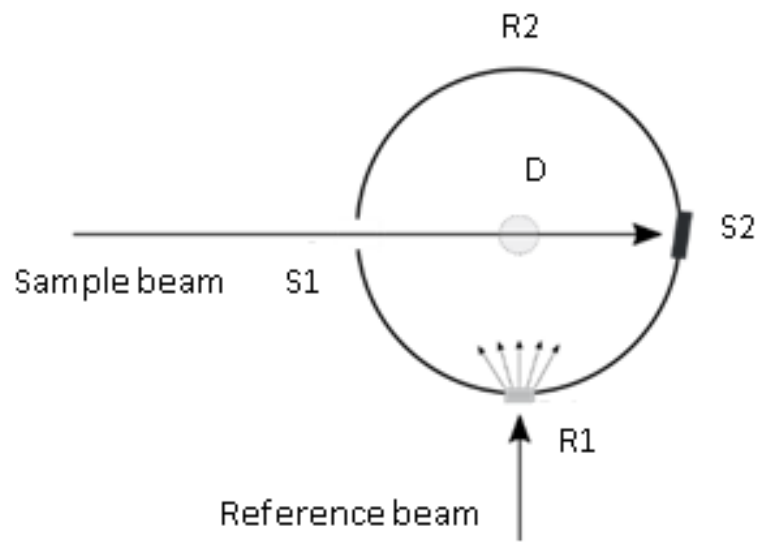

Figure 3 A schematic of the integrating sphere, reference and sample beam paths and measuring port locations (S1 and S2).

For the first and second measurement, port S1 was left opened and the Teflon cap was replaced by the cuvette with the sample at port S2. If the cuvette is tilted, the resulting signal $\left(I_{R+B S}\right)$ is proportional to the sum of reflected and backward scattered light power. If the cuvette was not tilted, the resulting signal $\left(I_{\mathrm{BS}}\right)$ is proportional to the backward scattered light power.

Once the two measurements mentioned above were acquired, reflectance spectrum was obtained as $R=I_{R+B S}-I_{B S}$. This reflectance will be needed later for the Kubelka-Munk equations to obtain some other optical parameters.

The absorption $(K)$, scattering $(S)$, extinction $(E=K+S)$ and scattering albedo $(\omega=S / E)$ coefficients were calculated by the Kubelka-Munk Theory [19] using the following equations: 
where $T$ is the ballistic transmittance and $d$ is sample thickness.

\section{Results}

\subsection{Particle size distribution}

Particle size distribution of the CB thermal oil-based sample was obtained by DLS technique using two equipments. This test had two goals: to obtain the particle size distributions of the nanoparticles suspended in the TH66 at different temperatures and to compare the two DLS systems in order to corroborate the appropriate set up of the new VASCO FLEX device.

Due to this proposed objective, particle size distributions of the samples in both equipment are presented in Figure 4 at room temperature:

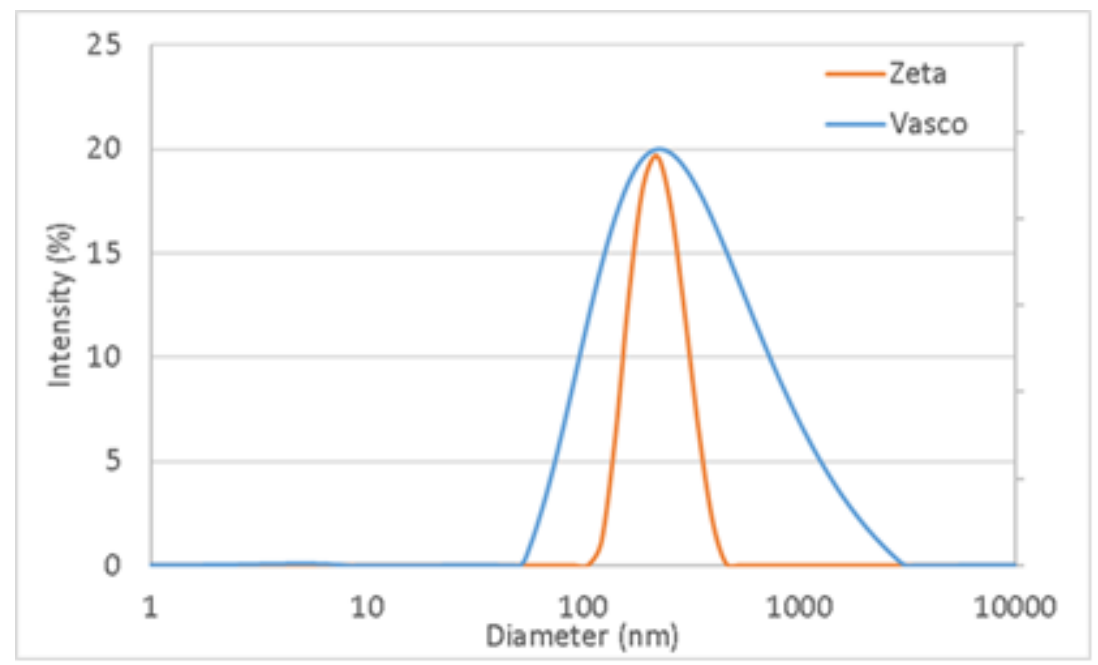

Figure 4 Particle size distribution of CB nanoparticles suspended in TH66 at room temperature in two different devices: Zetasizer Nano (orange line) and VASCO FLEX (blue line).

As it can be observed, distribution curves are centered on the same value, $200 \mathrm{~nm}$ approximately, at room temperature. It means that the size of the nanoparticles measured with both DLS systems are similar and therefore, both equipments are in concordance. 
Solar nanofluids are subjected to high temperatures in their real applications, consequently the behaviour of the nanofluid needs to be studied also at high temperature. In this study, measurements have been carried out at $85^{\circ} \mathrm{C}$ in both DLS systems to achieve two objectives: to know the influence of the temperature on the particle size distribution and to analyse the suitability of both DLS equipments at high temperature.

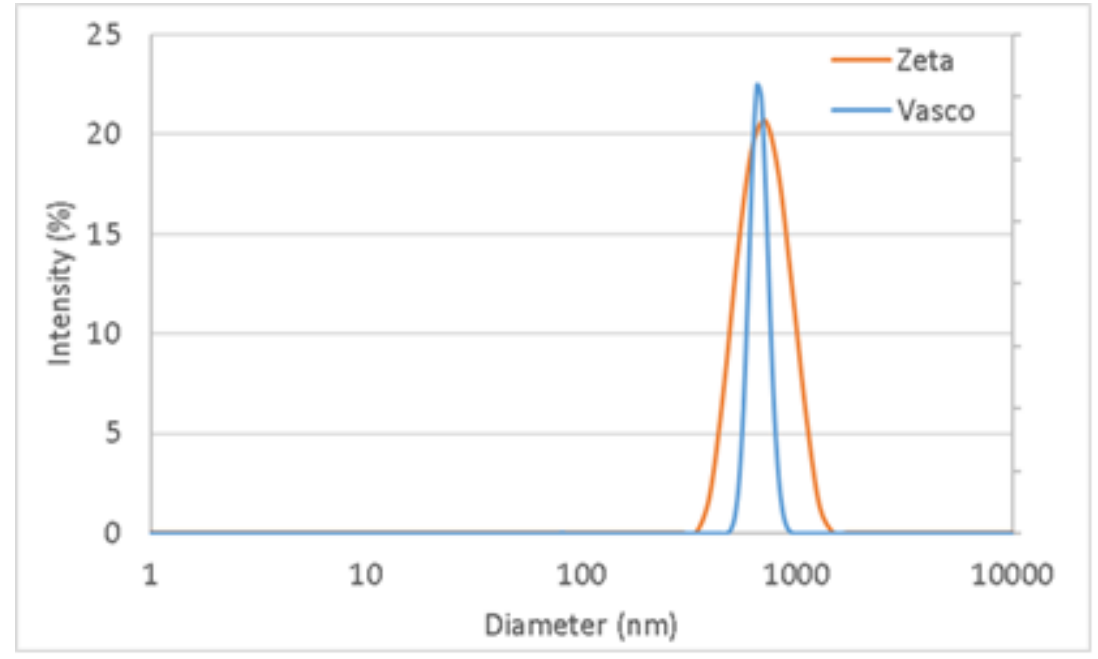

Figure 5 Particle size distribution of $\mathrm{CB}$ nanoparticles suspended in $\mathrm{TH} 66$ at $85^{\circ} \mathrm{C}$ in two different devices: Zetasizer Nano (orange line) and VASCO FLEX (blue line).

From the results obtained in Figure 5, it is possible to confirm the convergence of the size results in both DLS systems (Zetasizer and VASCO). Although the distributions are centered on similar values, this is different to that measured at room temperature (Figure 4). It can be concluded by comparing Figures 4 and 5 that both DLS equipments are in concordance regardless of the measuring temperature, but also that the characteristic particle sizes increase with temperature. The fact that particle size becomes bigger with the temperature is due to the agglomeration: nanoparticles aggregate forming clusters which diameter is greater than that of the dispersed nanoparticles.

In order to get a deeper study of the sample behaviour at high temperature, the sample was thermally treated at $85^{\circ} \mathrm{C}$ for 30 minutes in a stove. After this treatment, particle size distribution of the samples was measured again at room temperature and at $85^{\circ} \mathrm{C}$, obtaining the same mean diameter than for the untreated sample measured at $85^{\circ} \mathrm{C}$. Figure 6 clearly 
indicates that once the samples are thermally treated, the nanoparticles agglomerate and remain unchanged, without forming larger agglomerates and without settling.

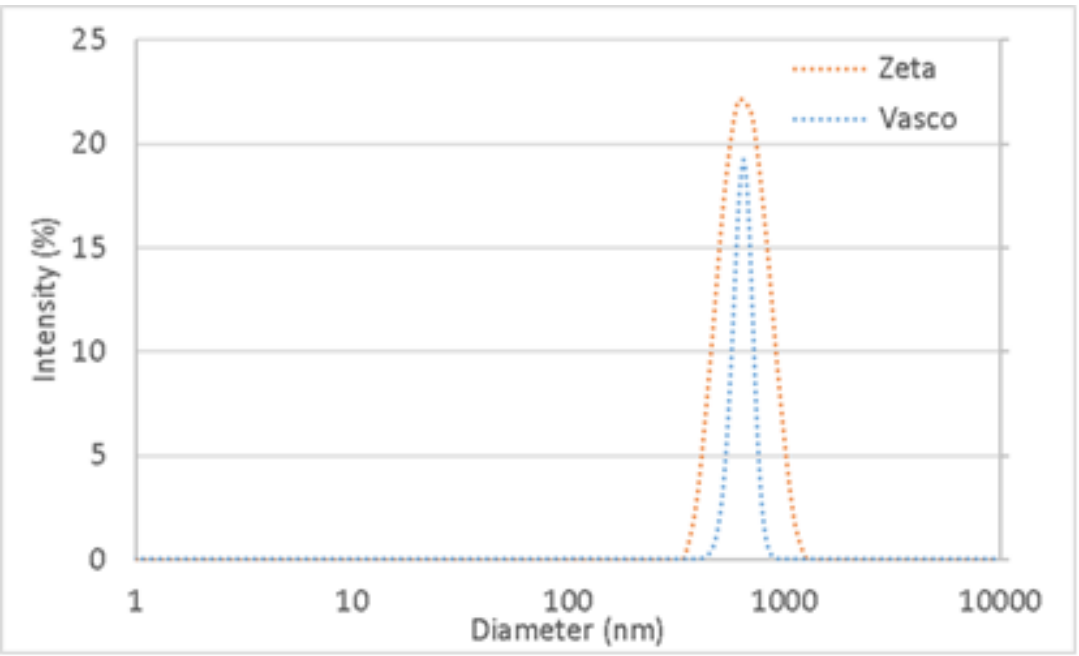

Figure 6 Particle size distribution of thermally treated CB nanoparticles suspended in TH66 at room temperature in two different devices: Zetasizer Nano (orange dotted line) and VASCO FLEX (blue dotted line).

Finally, the treated sample size was also measured at $85^{\circ} \mathrm{C}$ to ensure the reliability of the argument established after the results shown above.

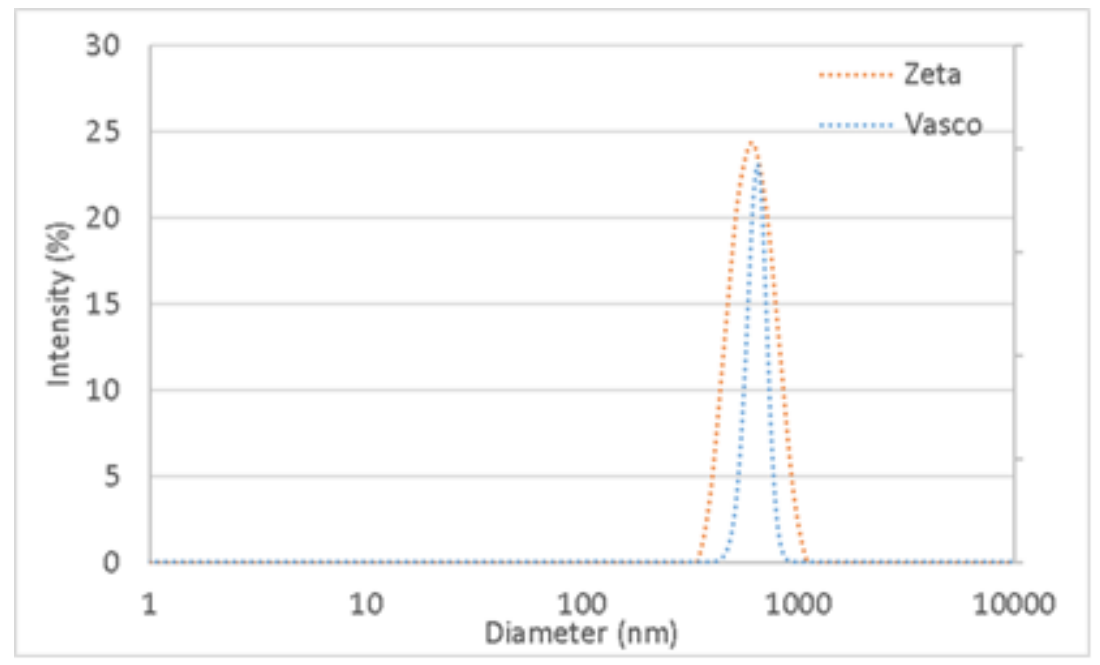

Figure 7 Particle size distribution of the thermally treated CB nanoparticles suspended in TH66 at $85^{\circ} \mathrm{C}$ in two different devices: Zetasizer Nano (orange dotted line) and VASCO FLEX (blue dotted line).

Indeed, once the sample has been treated, it remains stable and the diameter is the same independently of the temperature that is measured. 
In addition to particle size distribution, the samples' Zaverage values over time are presented. This is an intensity-based overall average size based on a specific fit to the raw correlation function data [20].

Zaverage values of each measurement have been compiled in Table 1 to have a better understanding about the particle size results.

Table 1 Zaverage of the samples before and after the thermal treatment measured at room temperature and $85^{\circ} \mathrm{C}$ in two different systems

\begin{tabular}{|c|c|c|c|c|}
\hline Temperature & \multicolumn{2}{|c|}{ Room Temperature } & \multicolumn{2}{c|}{$85^{\circ} \mathrm{C}$} \\
\hline & Zeta & Vasco & Zeta & Vasco \\
\hline Before thermal treatment & 210 & 310 & 666 & 600 \\
\hline After thermal treatment & 607 & 661 & 623 & 600 \\
\hline
\end{tabular}

It is extracted from Table 1 that the mean size of the CB nanoparticles before being treated and measured at room temperature is around $260 \mathrm{~nm}$. However, the averaged diameter after the nanofluid exposed to the thermal treatment measured at room temperature and $85^{\circ} \mathrm{C}$, and when it has not been treated but measured at $85^{\circ} \mathrm{C}$ is around $630 \mathrm{~nm}$. It clearly indicates that nanoparticles agglomerated due to the effect of the temperature. However, it is important to remark that in all cases (fresh and thermally treated) samples were homogeneously dispersed and no particle settle was observed, confirming the DS surfactant as appropriate for high temperature applications.

\subsection{Morphology}

Samples of the thermal oil-based carbon black nanofluids were observed by TEM. 

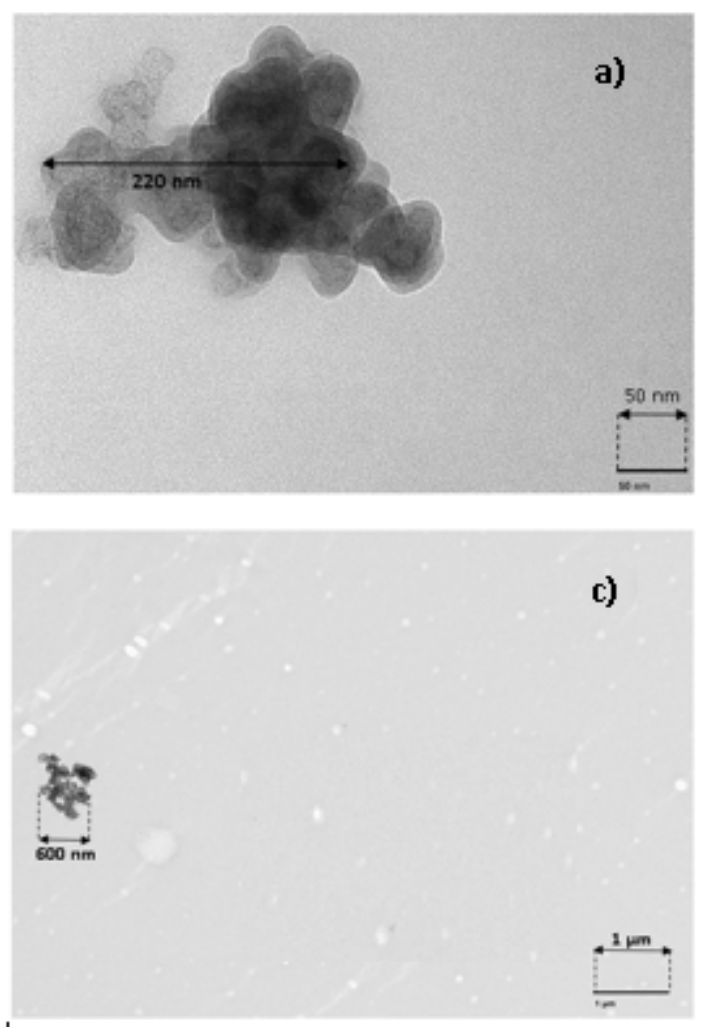
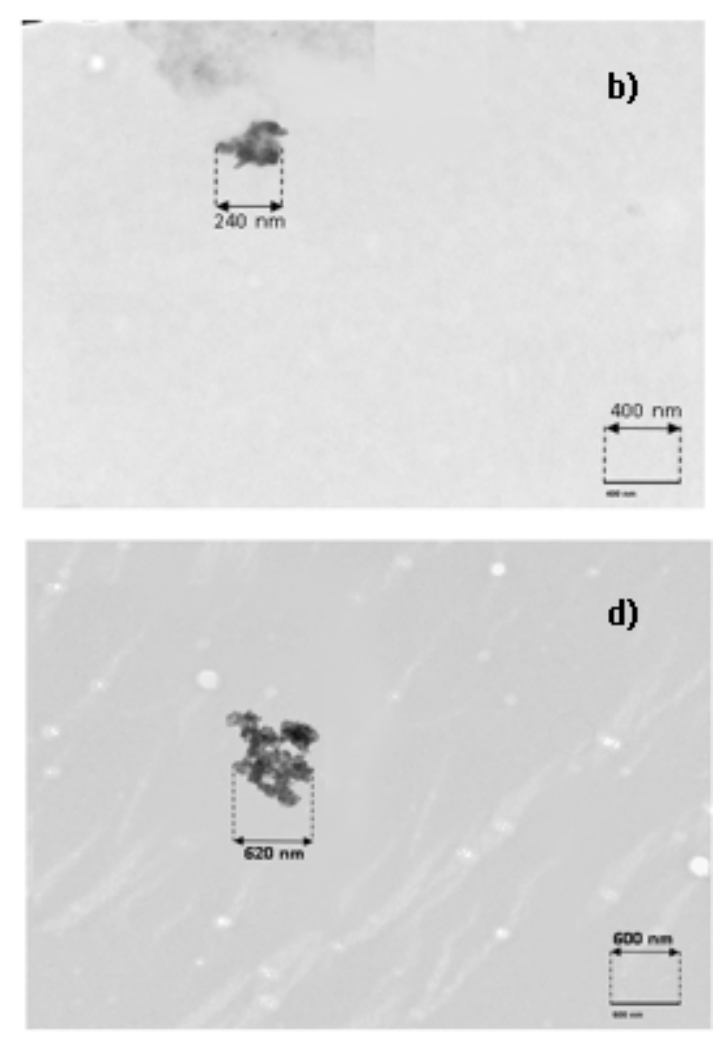

Figure 8 TEM images of a), b) TH66-based CB nanofluid before the thermal treatment and c), d) TH66-based CB nanofluid after the thermal treatment.

Figure 8 a) and b) present TEM images of the fresh nanofluid (before thermal treatment). It is possible observe after averaging the main sizes of different TEM images, that the mean diameter obtained is around $200 \mathrm{~nm}$. It is worth highlighting the similarity with the results given above by the two DLS equipment (Figure 4).

Figure $8 \mathrm{c}$ ) and d) show TEM images of the thermally treated nanofluid. It is observed that in this case the clusters are bigger and the mean diameter has increased with respect to the previous case. Some TEM images have been processed and the averaged size is around 600 $\mathrm{nm}$, matching up with the particle size distribution obtained from both DLS systems (Figure 6).

\subsection{Optical Characterization}

To have an initial idea of the optical properties of the base HTF (TH66) and the carbon black nanofluid (TCB), a comparative image of them is illustrated in Figure 9. Simple visual inspection 
can evaluate the difference between them: the base fluid is almost transparent with light yellow shade and after adding the CB nanoparticles the resulting nanofluid is black.

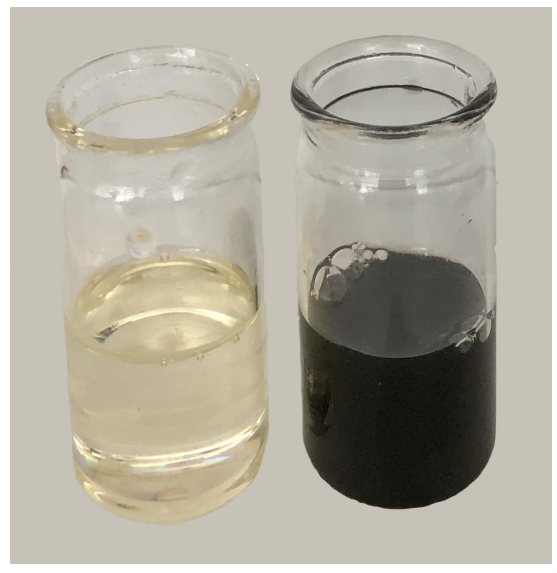

Figure 9 Therminol 66 (left) and Therminol 66 with carbon black nanoparticles (right).

Due to the importance of the optical properties of the studied nanofluids, different spectra were acquired in a spectrophotometer with and without integrating sphere in order to achieve a deeper optical study of the samples.

First of all, ballistic transmittance spectra for the base fluid and the nanofluid without integrating sphere was measured and it is shown in Figure 10.

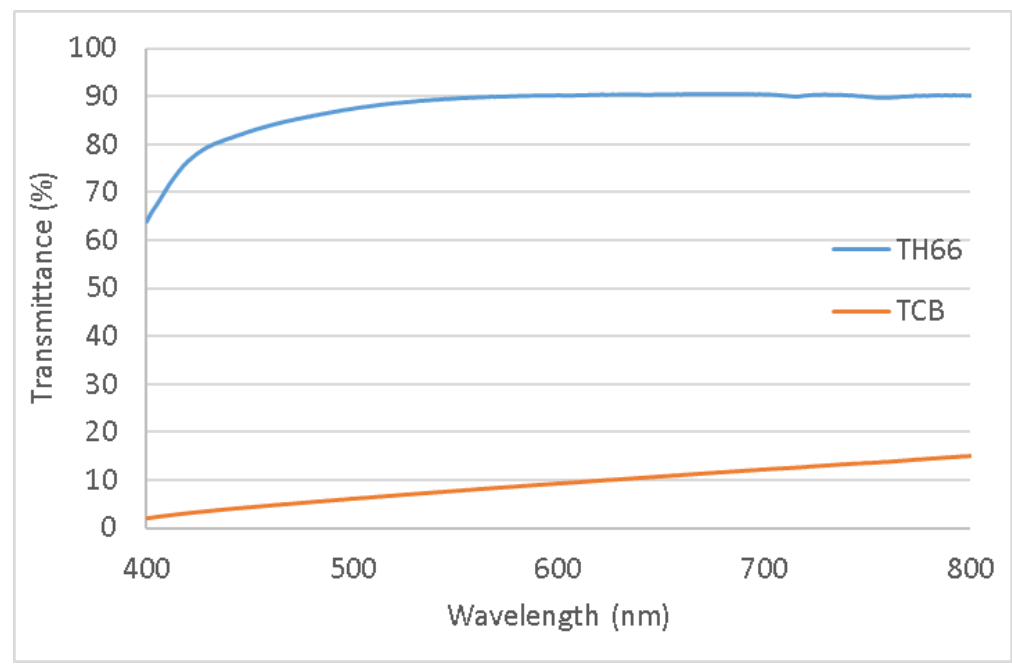

Figure 10 Ballistic transmittance of TH66 (base fluid) and TCB (nanofluid formed by TH66 and CB nanoparticles). 
It is clearly observable the considerably transmittance reduction that occurs when adding nanoparticles to the base fluid. This is an indicator of the potential of this nanofluid as solar absorber.

Apart from this parameter, it is important to study the absorption and scattering albedo coefficients, to ensure the suitability of the sample for its applications in solar energy technologies. Because of that, Kubelka-Munk theory was used and the coefficients mentioned above were calculated from the spectra acquired with the integrating sphere.

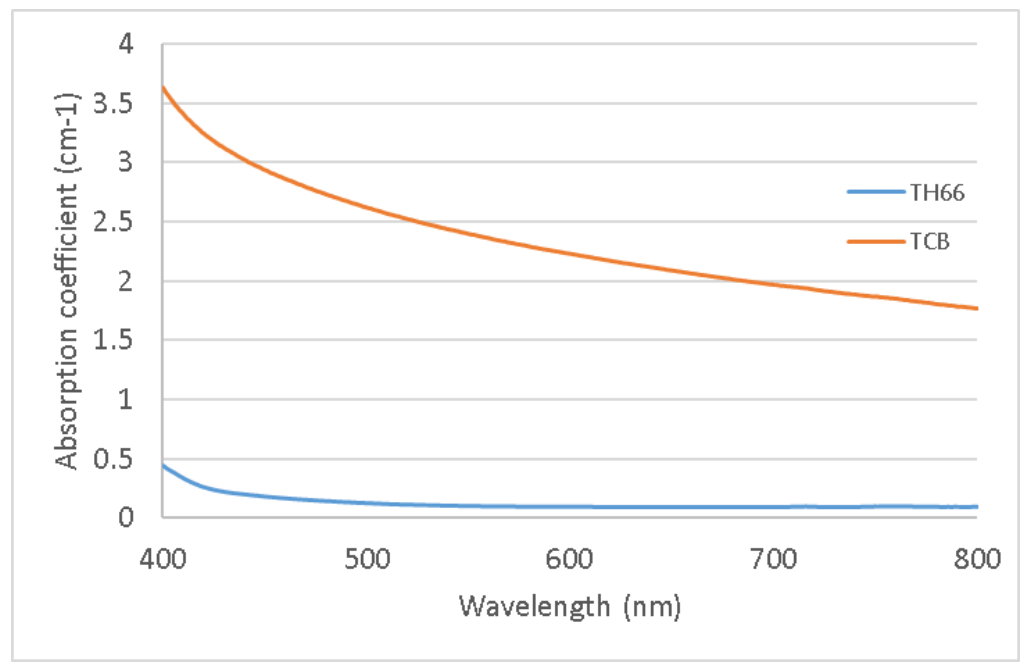

Figure 11 Absorption coefficient of TH66 (base fluid) and TCB (nanofluid formed by TH66 and CB nanoparticles) The absorption coefficient is one of the most important parameter for a nanofluid that has been proposed as solar absorber. It was obtained by means of equations 1, 2 and 3, in which 
there were included the spectrophotometer measurements with and without integrating sphere. As it was expected, it is extracted from Figure 11 that the absorption coefficient of the nanofluid is remarkable higher than that of the base fluid, presenting increments of about $800 \%$ by just adding a concentration of $0.0016 \%$ wt of CB nanoparticles.

Finally, the scattering albedo coefficient of the nanofluid was also calculated to evaluate the amount of light from the extincted one that is scattered by the nanoparticles and consequently, will not be absorbed. The results are shown in Figure 12.

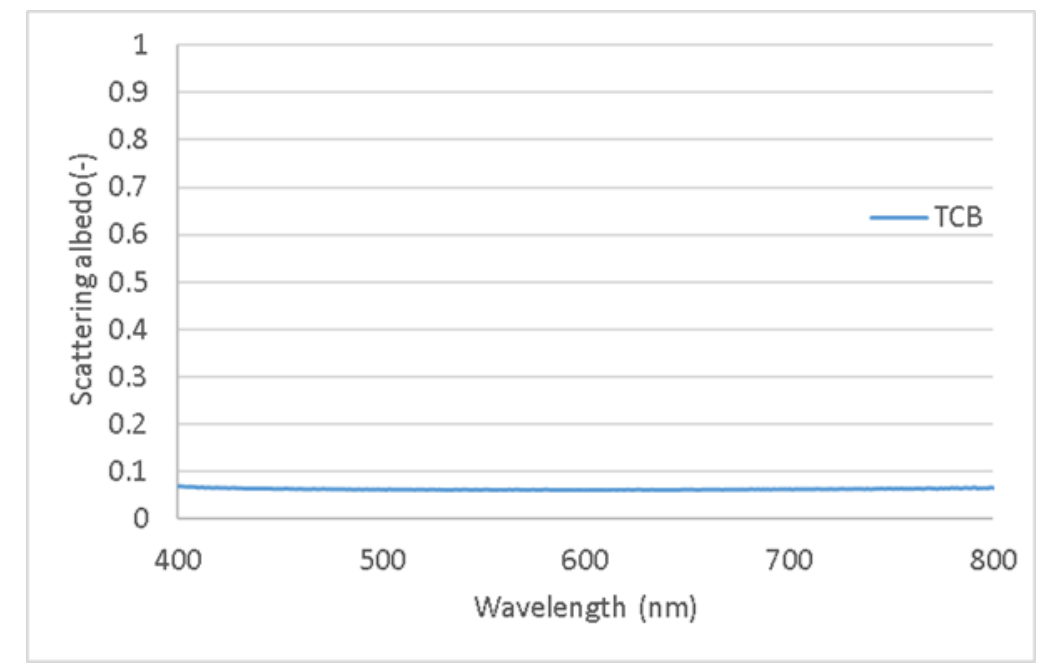

Figure 12 Scattering albedo of TCB (nanofluid formed by TH66 and CB nanoparticles) Scattering albedo values lower than 0.1 clearly indicates that most of the extinted light in this nanofluid is absorbed and less than $10 \%$ is scattered. The results are also in concordance with those previously shown in the literature [21 - 23].

\section{Conclusions}


A thermal oil-based nanofluid with CB nanoparticles in a concentration of $0.0016 \%$ wt and DS as surfactant in the same concentration has been syntethised and its particle size distribution were studied at room temperature and under high temperature conditions to observe its behaviour in conditions closer to those in real applications.

In order to measure the size particle distribution of the nanofluid at different temperatures, two DLS systems were used. One of the system is widely used in the literature, Zetasizer Nano, while the other one, VASCO FLEX, is a new external system that is suitable for hightemperature measurements.

Particle size distribution was evaluated at room temperature with Zetasizer and VASCO FLEX, with good results since both equipment were in concordance. Afterwards, the sample was thermally treated at $85^{\circ} \mathrm{C}$ for 30 minutes in a stove, and its particle size distribution was measured again. The obtained results were different to those obtained with the fresh samples, concluding that temperature plays an important role when working with this nanofluid. Once the nanofluid was thermally treated; bigger clusters were formed and remain even when reducing the temperature. Important also to highlight that the nanofluid remain stable even after the thermal treatment, highlighting that the used surfactant (DS) is suitable for high temperatures.

All the measurements before and after the thermal treatment and at different temperatures were performed in both DLS devices. The agreement obtained between both systems' results ensures the reliability of the measurements and equipment.

In terms of optical properties, it is possible to stablish this nanofluid as a good potential solar absorber. The absorption of the base fluid (in this case Therminol 66) is enhanced around $800 \%$ with a low load of nanoparticles, what makes the $C B$ very interesting nanoparticle for the solar energy applications. In addition, the scattering albedo is low, meaning that most of the 
extincted light is absorbed and that position this nanofluid in the top of the solar nanofluids market. 


\section{Abbreviations}

Concentrated Solar Power

(CSP)

Heat Transfer Fluid

(HTF)

Dynamic Light Scattering

(DLS)

Carbon Black

(CB)

Therminol 66

Therminol 66 with CB nanoparticles

Diphenyl sulfone

(DS)

Transmission Electron Microscopy

(TEM)

\section{Acknowledgements}

The authors gratefully acknowledge the financial support from Pla de Promoció de la Investigació a I'UJI (Project: UJI-B2016-47) from the Spanish Ministry of Economy and Competitiveness (Project: ENE2016-77694-R) and from the Generalitat Valenciana (Project: PROMETEU/2016/079).

\section{Author details}

${ }^{1}$ Department of Mechanical Engineering and Construction, Universitat Jaume I, Castellón, Spain

\section{Competing interests}

The authors declare that they have no competing interests. 


\section{References}

1. Philibert, C., 2011. Interactions of Policies for Renewable Energy and Climate 22.

2. Minardi, J.E., Chuang, H.N., 1975. Performance of a "black" liquid flat-plate solar collector. Sol. Energy 17, 179-183.

3. Otanicar, T.P., Phelan, P.E., Golden, J.S., 2009. Optical properties of liquids for direct absorption solar thermal energy systems. Sol. Energy 83, 969-977.

4. Burke, 1982. Thermal and Photochemical Studies of Solar Energy 6, 481-490.

5. Taylor, R.A., Phelan, P.E., Otanicar, T.P., Adrian, R., Prasher, R., 2011. Nanofluid optical property characterization : towards efficient direct absorption solar collectors. Nano. Res. Lett, 6:225.

6. R. Taylor, S. Coulombe, T. Otanicar, P. Phelan, A. Gunawan. 2013. Small particles, big impacts: A review of the diverse applications of nanofluids. J. Appl. Phys. 113:011301.

7. Karami, M., Bahabadi, M.A., Delfani, S., Ghozatloo, A., 2014. A new application of carbón nanotubes nanofluid as working fluid of low-temperature direct absorption solar collector. Sol. En. Mat. Cells 121, 114-118.

8. Lenert, A., Wang, E.N., 2012. Optimization of nanofluid volumetric receivers for solar thermal energy conversion. Solar Energy 86, 253-265.

9. Mahian, O., Kianifar A., Kalogirou, Pop I., Wongwises, S., 2013. A review of the applications of nanofluids in solar energy. Int. J. Heat and Mass Transf. 57, 582-594.

10. Xie, W.Y. and H., 2015. A review on nanofluids: preparation, stability mechanisms and applications. Int. J. Therm. Sci. 87, 228-240.

11. Wen, D. and Y.D., 2003. Effective thermal conductivity of Aqueaous Suspensions of 
Carbon Nanotubes (Carbon Nanotubes Nanofluids) . J. Therm. and Heat Transf 18, 481485.

12. Liu, X., Zhang, Z., Wu, Y., 2011. Absorption properties of carbon black/silicon carbide microwave absorbers. Composites: Part B 42, 326-329.

13. Sharif, M., Golestani, F., Khatibi, E., Sarpoolaky H., 2009. Dispersion and stability of carbon black nanoparticles, studied by ultraviolet-visible spectroscopy. J. Tai. Inst. Of Chem. Eng 40, 524-527.

14. Soares, M.C.F., Viana, M.M., Schaefer, Z.L., Gangoli V.S., Cheng, Y., Caliman, V., Wong, M.S., Silva, G.G., 2014. Surface modification of carbon black nanoparticles by dodecylamine: Thermal stability and phase transfer in brine medium. Carbon 72, 287295.

15. Kearney, D., Herrmann, U., Nava, P., Kelly, B., Mahoney, R., Pacheco, J., Cable, R., Potrovitza, N., Blake, D., Price, H., 2003. Assessment of a molten salt heat transfer fluid in a parabolic trough solar field. J. Sol. Eng. 125, 170-176.

16. Vignarooban, K., Xu, X., Arvay, A., Hsu, K., Kannan, A.M., 2015. Heat transfer fluids for concentrating solar power systems- A review. Appl. En. 146, 383-396.

17. Gimeno-Furio, A., Navarrete, N., Martinez-Cuenca, R., Julia, J.E., Hernandez, L., 2018. Influence of high temperature exposure on the thermal and optical properties of thermal oil-based solar nanofluids. J. Nanofluids 7, 1-8.

18. Gimeno-Furio, A., Navarrete, N., Mondragon, R., Hernandez, L., Martinez-Cuenca, R., Cabedo, L., Julia, J.E. 2017. Stabilization and characterization of a nanofluid based on a eutectic mixture of diphenyl and diphenyl oxide and carbon nanoparticles under high temperature conditions. Int. J of Heat and Mass Transf. 113, 908-913.

19. Jaradat, A., Ali, M., Al-Akhras, Makhadameh, G., Aljarrah, K. 2011. Artificial semi-rigid 
tissue sensitized with natural pigments: Effect of photon radiations. J Pharm Bioallied Sci. 3:266-276.

20. M. Instruments. 2011. Inform white paper dynamic light scattering. pp. 1-6.

21. Sani, E., Mercatelli, L., Barison S., Pagura, C., Agresti, F., Colla, L., Sansoni, P. Potential of carbon nanohorn-based suspensions for solar thermal collectors. 2011. Sol. En. Mat. Sol. Cells. 95, 2994-3000.

22. Wagner, T.R., Houf, W.G., Incropera, F.P., 1980. Radiative property measurements for india ink suspensions of varying concentration. Sol. Energy 25, 549-554.

23. Mercatelli, L., Sani, E., Giannini, A.,Di Ninni, P., Martelli, F., Zaccanti, G., 2012. Carbon nanohorn-based nanofluids: Characterization of the spectral scattering albedo. Nanoscale Res. Lett 7, 1-12. 